\title{
Toward the Semiclassical Theory of High Energy Heavy Ion Collisions
}

\author{
E.V.Shuryak \\ Department of Physics and Astronomy, \\ State University of New York, Stony Brook NY 11794-3800
}

\begin{abstract}
Sudden deposition of energy at the early stage of high energy heavy ion collisions makes virtual gluon fields real. The same is true for virtual vacuum fields under the topological barrier, excited to real states at or above the barrier, gluomagnetic clusters of particular structure related to the sphalerons of the electroweak theory. Semiclassically, these states play the role of the "turning points". After being produced they explode into a spherical shell of coherent field which then turn into several outgoing gluons. Furthermore, this explosions promptly produce quark pairs, as seen from explicit solution of the Dirac equation. The masses of such clusters depend on their size, and are expected to peak at $M \sim 3 \mathrm{GeV}$. After we briefly review those concepts in a non-technical manner, we discuss what observable consequences the production of such clusters would make in the context of heavy ion collisions, especially at the RHIC energies. We discuss entropy and especially quark production, event-by-event fluctuations in collective effects like radial and elliptic flows and $J / \psi$ suppression. Coherent fields and their geometry increase the jet quenching, and we also point out the existence of "explosive edge" which jump-start collective effects and may affect unusual phenomena seen at RHIC at large $p_{t}$.
\end{abstract}

\section{Introduction}

This work ${ }^{1}$ consists of two very different parts. Section 2 is brief non-technical review of the semiclassical theory of high energy collisions, covering recent progress described in details in more technical works. (We think it is really necessary, and helpful for the readers, to present some coherent picture first: although most concepts are not new they were mostly developed not in the context of QCD, and certainly not for heavy ion collisions.) Its only new part is section 2.2 , in which the main idea is demonstrated by a simple quantum-mechanical toy model, treated without any approximations.

The main body of the paper includes applications of these ideas to heavy ion collisions. In section 3 we discuss why we think they are relevant for the initial stages of the collisions, especially at RHIC energies. We continue with discussion of jet quenching and phenomena at the edge of the system in section 4 , ending with possible manifestations of clusters in event-by-event fluctuations in section 5 .

${ }^{1}$ Its early version exist as a preprint and a talk at Quark Matter 2002 [1]. 


\subsection{Tunneling and related excitations in QCD}

The semiclassical theory of the tunneling phenomena in the QCD ground state, associated with topology structure of the Yang-Mills fields is based on the so called instanton solutions $[2,3]$. It is by now understood in significant detail and has strong ties to hadronic phenomenology and lattice studies. These phenomena are known to play an important role in chiral symmetry breaking, hadronic spectroscopy, form-factors etc, see e.g.[4] for a review.

The semiclassical approach to various high-energy reactions is much less developed. It had started in the early 1990 [5, 6], when baryon-number violating tunneling in the electroweak theory has been actively discussed. It waned several years later when it became clear that those fascinating phenomena cannot be experimentally observed.

In a very crude way, one may say that collision of two (virtual) gauge bosons, $\mathrm{W}, \mathrm{Z}$ in electroweak theory or gluons in QCD, can produce two type of objects shown in Fig.1(b), a single on-shell gauge boson or a turning state cluster . The former is the so called Lipatov vertex, the main ingredient of the BFKL ladder [33] providing high energy asymptotic behavior in pQCD. The latter is the process to be discussed in detail below.

The first QCD effects of similar origin discussed were instanton-induced multi-jet production: the search in this direction continues at HERA, see recent review in [7]. However, due to large scale involved and small-size instantons, this phenomenon is also associated with small cross sections, and even if the predicted signal be found it would not be easy to prove that it is not due to some perturbative diagrams.

The situation is different at the so called semi-hard scale $Q \sim 1-2 \mathrm{GeV}$, at which tunneling phenomena are in some cases so large than they simply dominate the contribution from the perturbative processes. Recently it was suggested that instanton-induced processes may explain why the cross section of high energy hadronic scattering grows with energy [10, 8, 9]. The reason is prompt production of specific hadrons (such as the scalar glueballs) and the topological clusters to be discussed in this work. Specific properties of the so called "soft pomeron" (such as its intercept and the slope) have been calculated with rather reasonable results. As unexpected qualitative insights found was a possible reason why there is no odderon in this theory [9]). New experimental test were suggested such as glueball production rate [10].

What made the situation qualitatively different now is a real breakthrough in understanding of the nature of semiclassical path including the structure of the turning states and its real (Minkowskian) evolution. We will describe those in the next section. 


\subsection{Confronting the RHIC puzzles}

Let us now switch to brief overview of heavy ion physics. Relativistic Heavy Ion Collider (RHIC) project have been completed in 2000 and made its first full-scale run with AuAu at full energy in 2001. The aim of it was to detect a transition of the QCD vacuum to qualitatively new phase, a deconfined and chirally symmetric Quark-Gluon Plasma (QGP) [12]. Among the predicted signals for it were robust collective phenomena known as radial and elliptic flows [13]. Already the first data from RHIC have quantitatively confirm these predictions, with equation of state consistent with that expected from QGP. Excellent agreement of all spectra goes till $p_{t} \sim 2 \mathrm{GeV}$, or for about 99.5 percent of all particles. (At higher $p_{t}$ one observed strong deviations from the naive parton model: we will return to this in section 4). Studies of correlations between $\pi K$ or $K N$ agree with significant time difference $\sim 5-6 \mathrm{fm} / \mathrm{c}$ between emission of $K$ and $\pi, N$. Event-by-event fluctuations are small and roughly consistent with thermal ones at freezeout. Composition of all secondaries agrees well with thermal production rates. All of it confirm that strong interaction in the system starts at time $\sim 1 / 2 \mathrm{fm} / \mathrm{c}$ and ends at $\sim 10-15 \mathrm{fm} / \mathrm{c}$, which means that the QGP is actually created at RHIC.

Let me explain a bit how the lower limit on this time was obtained. The elliptic flow ${ }^{2}$ is characterized by the parameter $\left.v_{2}=<\cos (2 \phi)\right\rangle$. It is is present only for non-central collisions, in which the overlap region of two nuclei have an almond-type shape shown in Fig.1(a). As we already mentioned in the introduction, hydrodynamics describes $v_{2}\left(p_{t}\right)$ well into the tails of particle spectra, up to $p_{t} \sim 2 \mathrm{GeV}$. At the same time other approaches - such as string-based or mini-jet-based ones - have problems reproducing growing $v_{2}\left(p_{t}\right)$.

The crucial point is that short initiation time $t_{i} \sim 0.5-1 \mathrm{fm} / \mathrm{c}$ is absolutely necessary in order to account for large observed $v_{2}$. If a period of "free streaming" of partons would exist for longer time, it is impossible to recover ellipticity of the shape. Moreover, following the observed impact parameter dependence of $v_{2}$ one finds that it peaks at large impact parameter $b \sim 8-10 \mathrm{fm}$, for which the width of the almond in Fig.1(a) is very small. And still all the hydro predictions apparently work, forcing us to conclude that the mean free path and equilibration time are much shorter than this width.

The main issue now is to understand how it happens, what is the dynamics responsible for QGP formation in a very such short time. Let me put this question as the first in the list of the RHIC puzzles which remains basically unexplained

- Early formation of significant pressure leading to explosive collective behavior

\footnotetext{
${ }^{2}$ A special role of elliptic flow stems from the fact that it allows to separate the initial-state (e.g. parton re-scattering) from the final-state interactions, since the direction of event impact parameter is unknown prior to the collision.
} 

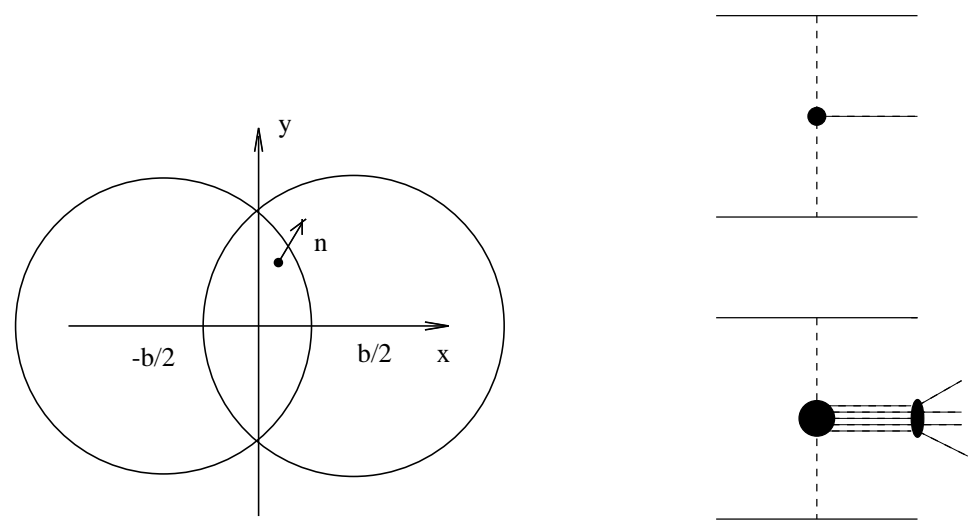

Figure 1: (a) The almond-shape overlap region for the non-central nuclear collisions with impact parameter $b$. (b) Two generic vertices producing either a physical gluon or a topological cluster from two colliding virtual gluons in a high energy collision.

- Large deficit of large- $p_{t}$ hadron yields, or jet quenching

- Large (and approximately $p_{t}$ independent) azimuthal asymmetry at large$p_{t}$

- Large baryon/meson ratio at large- $p_{t}$, much larger than in the usual jet fragmentation

Those are only questions directly related to data, but there are many more issues of crucial importance which we have to answer, such as: at what time quarks appear, is excited matter produced gluonic or closer to equilibrium QGP?

\subsection{Theory overview}

To put these ideas in proper context, recall that initial stages of high energy heavy ion collisions have been for a long time associated with re-scattering of partons with momenta about 1-2 GeV (later called mini-jets) considered already in [12]. The parton cascade model by Geiger and Muller [14] have added "branching" of virtual partons, or bremsstrahlung. Important role of pQCD processes of 2-to-n type has been discussed in [15]. Recent development along the pQCD line [19] included account for Landau-Pomeranchuck-Migdal effect and other improvements. Although perturbative equilibration was found possible, its rate is very slow, and basically no collectivity was predicted by the mini-jet models like HIJING. Furthermore, it was nicely quantified in [16] by 
how much pQCD-based scenario with 1-2 GeV cutoff misses what is needed: in order to reproduce the elliptic flow by a parton cascade the product of the gluon density ${ }^{3}$ times the cross section should be increased by a factor of about 80. So, naive extrapolation of pQCD to momenta $Q \sim 1 \mathrm{GeV}$ have failed to reproduce RHIC data. This has created a serious challenge to the theory.

Significant attention has been attracted by the idea stemming from a possible saturation [20] phenomenon at small $\mathrm{x}$, which get some support by HERA data on deep inelastic scattering. It was further argued that when, at sufficiently small $\mathrm{x}$, the gluon occupation numbers reach the magnitude $\sim O\left(1 / \alpha_{s}\right)>>1$ the classical approach to YM field becomes possible [21]. Such matter was called the Color Glass Condensate (CGC), and its spontaneous "materialization" may significantly contribute to the QGP formation, as recent numerical studies using classical Yang-Mills equation have shown [22].

We will argue in this work that the CGC is not just transverse classical field, but a part of it possesses topological properties. In terms of the filed strength it means that the electric and magnetic field strength are not orthogonal, $\vec{E} \vec{B}$ is substantial, which leads to quark/chirality production due to chiral anomaly. We will show that the energy range of RHIC provides an especially good window of opportunity to see fascinating phenomena related to topological tunneling, producing a spectacular "firework" of exploding topological clusters.

Recently first steps were made toward the understanding of the quite different limit, the strong coupling limit of the QGP phase. Specifically the so called 't Hooft coupling is assumed to be large $g^{2} N_{c}>>1$, and indeed in QGP produced at RHIC, with $T=(1-3) T_{c}$ this seems to be the case. The calculations are done via the famous $\mathrm{AdS} / \mathrm{CFT}$ correspondence for $\mathcal{N}=4$ supersymmetric Yang-Mills theory. In it the coupling does not run and one can study the theory at any value. Let me mention only one paper of the kind [18] focused on shear viscosity at high $\mathrm{T}$. Their main result is the following shear viscosity coefficient

$$
\eta=\frac{\pi}{8} N_{c}^{2} T^{3}
$$

which is significantly smaller than the weak coupling results $\eta \sim N_{c}^{2} T^{3} / \alpha_{s}^{2} \log \left(\alpha_{s}\right)$, [17]. If confirmed that these results can be used for QCD, it would mean that the mean free path is so short that one can use ideal hydrodynamics, but probably not a parton cascade.

\section{Quantum Mechanics of the Yang-Mills fields}

\subsection{Topological coordinates and the tunneling paths}

In this section we make brief review of the general setting, aimed at nonspecialists and explaining the main ideas and vocabulary used.

\footnotetext{
${ }^{3}$ Note however, that the multiplicity of partons cannot exceed that of final hadrons, because of quite fundamental limitation: the entropy can never decrease.
} 
Quantization of YM fields is simpler in the $A_{0}=0$ gauge, in which the canonical momentum is electric field " $\vec{P} "=\frac{d \vec{A}}{d t}=\vec{E}$ and the electric part of the energy $\vec{E}^{2}$ is identified as the kinetic term. The nonlinear magnetic term is identified as a potential energy: so schematically YM can be viewed as many coupled non-linear oscillators with a potential of the type $\vec{B}^{2} \sim\left(A^{2}+A^{3}+A^{4}\right)$.

A specific combination of $A_{\mu}$, called the Chern-Simons number

$$
N_{C S}=\int d^{3} x K_{0}
$$

is a special coordinate related to the so called topological current $K_{\mu}$.

$$
K_{\mu}=-\frac{1}{32 \pi^{2}} \epsilon^{\mu \nu \rho \sigma}\left(\mathcal{G}_{\nu \rho}^{a} \mathcal{A}_{\sigma}^{a}-\frac{g}{3} \epsilon^{a b c} \mathcal{A}_{\nu}^{a} \mathcal{A}_{\rho}^{b} \mathcal{A}_{\sigma}^{c}\right)
$$

The potential energy of the Yang-Mills field versus this coordinate is schematically shown in Fig.2: it is the periodic function, with zeros at all integer points. Those are called "classical vacua", they have zero fields but non-zero and topologically distinct $A_{m}^{a}$. The QCD vacuum is a quantum superposition of ground states near all of those classical vacua with the same amplitude ${ }^{4}$.

The natural language to describe quantum mechanical tunneling is Feynman path integral formulation. The tunneling can be described by specific paths, which start at one minimum of the potential and end up in another. The path with the minimal action is called the instanton, it satisfies classical equations of motion in Euclidean time and dominates the Feynman integral. The line on the bottom of that figure is such an instanton (shown by the lowest dashed line): it corresponds to the zero energy solution. The corresponding (Minkowskian) action $S_{\text {inst }}$ is imaginary and thus the tunneling probability is $\sim \exp \left(-\left|S_{\text {inst }}\right|\right)$. All this has been known since mid-70's.

In this paper we focus on other paths shown in this Figure. At the moment of high energy collisions a sudden localization of all quantum coordinates including the topological one takes place. Although their values remain about the same as they were prior to the collision moment, the system suddenly get placed at or above the barrier ( this case is shown by the dashed line (a) in Fig.2). Similar phenomenon is well known perturbatively: the partons (virtual field harmonics of the target or projectile) after collisions becomes real outgoing radiation. The same phenomenon happens for non-perturbative virtual fields as well: therefore some of the turning states we will discussed below are remnants of the interrupted instantons, already present in the vacuum.

Another possibility ( shown by the dashed line (b) in Fig.2) is that a system at the collision moment is not under barrier, but becomes able to tunnel through it because it gets excited enough. The corresponding amplitude would include exponential factors (as all tunneling amplitudes), but not some of the

\footnotetext{
${ }^{4}$ Other states exist also, but in those strong interaction would have a non-zero $\mathrm{CP}$ violation excluded experimentally.
} 
pre-exponential ones (such as quark condensates) which are included in the "instanton density" of the QCD vacuum. Whatever way the system is driven, it emerges from under the barrier via what we will call "a turning state", familiar from WKB semiclassical method in quantum mechanics.

The gluonic clusters to be discussed in this work are such turning states of the QCD paths. Those are relatives of the so called sphaleron ${ }^{5}$ solution of electroweak theory. This is a point where the path crosses the barrier and total energy is equal to only potential one. From there starts the real time motion outside the barrier. Here the action is real and $\left|e^{i S}\right|=1$. That means that whatever happens at this Minkowski stage has the probability 1 and cannot affect the total cross section of the process: this part of the path is only needed for understanding of the properties of the final state.

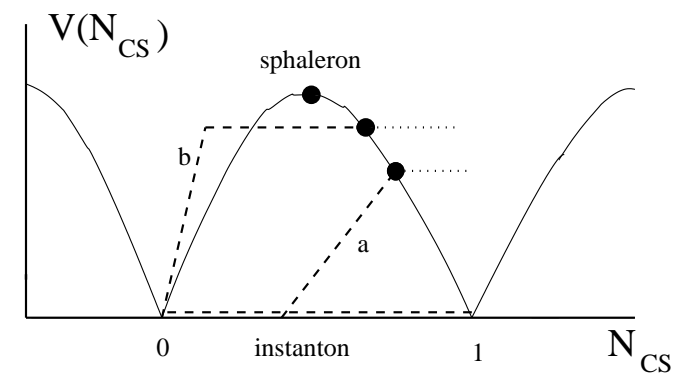

Figure 2: Schematic plot of the energy of Yang-Mills field versus the ChernSimons number $N_{c s}$. It is a periodic function, with zeros at integer points. The instanton (shown by the lowest horizontal dashed line) is a transition between such points. However if some nonzero energy is deposited into the process during transition, the virtual paths (the dashed lines) emerges from the barrier, via the turning points (black circles). The later real time motion outside the barrier (shown by horizontal dotted lines) conserves energy, as the driving force is switched off. The maximal cross section corresponds to the transition around the top of the barrier, the sphaleron.

\subsection{Exciting a quantum system from under the barrier}

Let me supplement the theoretical ideas in the field theory context by a simple quantum-mechanical example of related phenomenon, which anybody can test without much difficulty. The setting can be any problem with a barrier and tunneling, for example the very often used double well potential

$$
V=\lambda\left(x^{2}-f^{2}\right)^{2}
$$

\footnotetext{
5 "Ready to fall" in Greek, according to Klinkhammer and Manton [23].
} 


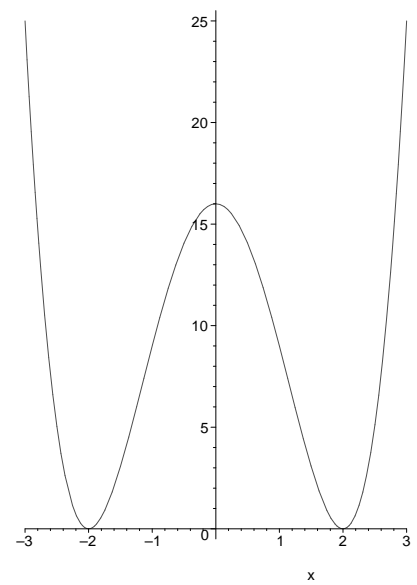

Figure 3: The double-well potential used.

in which a particle of $\mathrm{m}=1$ is placed. For the parameters $\lambda=1, f=2$ it is plotted in Fig.3. The value of $f$ is selected since the maximum of the potential $V(0)=16$ (the "sphaleron mass" of this problem) makes about the same number of oscillator quanta in a well as in QCD applications.

The ground state wave function is well familiar to everybody, it has two maxima corresponding to both wells, at $x \approx \pm f$, with relatively small probability below the barrier, at $x \sim 0$.

The question we would like to ask is what happens if one rapidly localizes the quantum particle under the barrier. One may view it pictorially as a narrow beam of particles localized near $\mathrm{x}=0$ and able to excite the system. More specifically, we are interested in the final states arising from such an experiment.

To answer those questions, let us introduce an external periodic perturbation acting on the system

$$
\delta V(x, t)=f(x) \exp [-i t \omega]
$$

with $f(x)$ well localized under the barrier, at $x \sim 0$. The specific shape of $f(x)$ does not matter as soon as it does not extends to the wells, where the ordinary oscillation quanta (analogs of gluons) can be excited. I have used several of them and will show results for $f=\exp \left(-4 x^{2}\right)$ and $f=1,|x|<.2 ; f=0,|x|>.2$.

The time dependence can be tuned to excite the n-th level $\omega=\left(E_{n}-E_{0}\right)$, and then the excitation probability

$$
P_{n} \sim|<0| f(x)|n>|^{2}
$$

be calculated directly from numerically calculated wave functions and energies. For even excitation functions $f(x)$ used, only even levels $\mathrm{n}=2,4$ etc can be excited. 
The result of the calculation is shown in the Fig.4(a). Note the strong peaking at the excitation equal to the "sphaleron mass" $\omega \approx 16$, indicating that the main final state is sitting at the barrier top. The reasons for the peak are as follows. For energies much less than $V(0)$ both the ground and the final wave functions are small under the barrier. For energies well above it $\psi_{n}(x)$ is not small but rapidly oscillating, so that its overlap with $f(x) \psi_{0}(x)$ is small.

Can one calculate the cross section for sphaleron production semiclassically in the field theory context?

We have already mentioned in the Introduction that the calculation of the cross section from first principles is not yet available. The original semiclassical approach with vacuum (undeformed) instantons was pioneered by Ringwald and Espinosa [5], who noticed that multi-gluon production is more (not less as in pQCD) probable than few-gluon one. Unfortunately it is good only at low energies of partonic sub-collision, much below the sphaleron mass $(10 \mathrm{TeV}$ in electroweak theory and $3 \mathrm{GeV}$ in $\mathrm{QCD}$ ). The problem to find a general semiclassical answer was known as the so called holy grail problem. Three methods toward its solution have been proposed

(i) Unitarization of the multi-gluon amplitude when it becomes strong was first suggested by Zakharov and worked out by Shifman and Maggiore [5]. Basically one can treat a sphaleron as a resonance, and even the resulting expression for the cross sections in [9] looks similar to Breit-Wigner formula. This is the most worked out approach, but it still cannot guarantee the parametrically accurate numerical values of the cross sections.

(ii) Landau method with singular instantons was applied by Diakonov and Petrov [6] (following some earlier works which are cited there) who were able to find the opposite limit of high energies. It follows from the comparison of the two limits, that the peak is indeed very close to the sphaleron mass, and the cross section is very close to be first order in instanton diluteness, not the second order as the initial probability. Unfortunately they were not able to find the solution at intermediate times which would provide the turning points of this approach.

(iii) Classical solution on the complex time plane [24] is another possible direction, in which a zig-zag shaped path in complex time includes classical evolution and tunneling in one common solution. Unfortunately, this interesting idea also has not been fully implemented, even for toy models with only scalar fields considered in this paper.

It would not be possible to describe here those approaches in any more detail. Let me just show the figure Fig.4 from the paper [25]: it shows the low energy and high-energy approximations for gluon production, calculated following "Landau method" mentioned above. Note strong resemblance to the quantum-mechanical excitation curves shown in the Fig.4(a). 

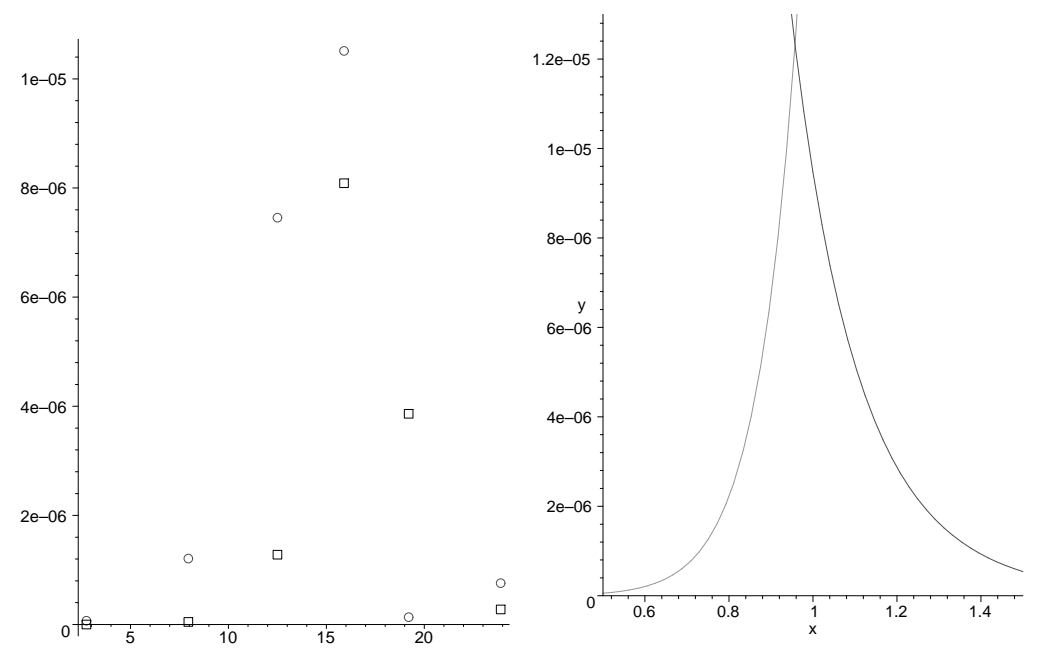

Figure 4: (a) The excitation probability $P_{n}$ of the double-well system versus the excitation energy. Two sets of points are for two excitation functions $f(x)$ mentioned in the text. Note that the peak excitation energy corresponds to the maximum of the potential, $V \approx 16$. (b) The inclusive gluon cross section of the process $g g \rightarrow$ sphaleron $\rightarrow g+\ldots$ versus the energy, in units of the sphaleron mass $x=Q / M_{s}$, from [25].

\subsection{The turning states}

Static gluonic turning states has been studied in a recent work [27] using two different approaches, and their structure has been determined. Without technical details, we give here some of their properties.

The first part of that work used the $\bar{I} I$ configurations. Those can be seen in two different ways. The traditional one is that such paths describe virtual processes in which the path goes under the barrier but eventually ends up in the original minimum, without tunneling. Another view ( adopted in [27]) is that such paths would rather be a time history repeated twice, with positive and negative times being mirror images of each other. If so, it can be seen as the probability (rather than the amplitude) of the vacuum excitation by some external current

$$
P \sim|<0| j_{\text {ext }} \mid \text { turning state }>\left.\right|^{2}
$$

The amplitude and its conjugate meet in the middle, which we will describe by the $t=0$ plane, also known as the "unitarity cut". This is the time moment when the turning states are born and released into real (Minkowski) space-time.

We will proceed directly to the second method, constrained minimization. Classical Yang-Mills is scale invariant, its energy can always be changed by re- 
scaling of the coordinates. However, the energy times the r.m.s. radius $E * R$, in which $\mathrm{R}$ can be defined as

$$
R^{2}=\frac{\int d^{3} r r^{2} B^{2}}{\int d^{3} r B^{2}}
$$

is invariant under scale transformation.

The corresponding turning states can thus be obtained [27] from the minimization of the potential energy of a static Yang-Mills fields, consistent with two appropriate constraints:

(i) fixed value of (corrected) Chern-Simons number $N_{C S}$ (2).

(ii) fixed value of the r.m.s. size $R$ (8).

To find those one should search for the minimum of the following functional

$$
E_{e f f}=\frac{1}{2} \int B_{m}^{2} d^{3} x+\frac{1}{\rho^{2}} R^{2}\left(A_{\mu}\right)+\kappa N_{C S}\left(A_{\mu}\right)
$$

where $1 / \rho^{2}, \kappa$ are two Lagrange multipliers. Although these two terms append YM equations and make it more complicated, an analytical solution is found. Skipping the details, let me only say that it is a well-localized magnetic ball, with $\vec{B}^{2}$ depending on the radial coordinate only. It is a $\mathrm{SU}(2)$ object, which means that it has 3 (out of 8) gluonic magnetic fields, with their field lines being closed in circles and rotating around axes x,y,z respectively.

The total energy of it times the size depend on kappa as follows

$$
E \rho=3 \pi^{2}\left(1-\kappa^{2}\right)^{2} / g^{2}
$$

while the Chern-Simons number is

$$
\tilde{N}_{C S}=\operatorname{sign}(\kappa)(1-|\kappa|)^{2}(2+|\kappa|) / 4
$$

Eliminating $\kappa$, one finds the profile of the topological potential barrier shown in Fig.2. Its maximum (to be called the YM sphaleron) corresponds to $\kappa=0$, its energy is $3 \pi^{2} /\left(g^{2} \rho\right)$ : the true potential however is exactly symmetric around $N_{c s}=1 / 2$.

\subsection{Explosion of the turning states}

After one knows the turning states, we can study their fate by solving the YM equation [27] starting from them. Of course, when field become weak enough those equations are no longer valid. We return to the question where it happens in section 3.2 .

The first study of the kind has been made a decade ago in electroweak theory [28] for the sphaleron, where it has been found that it decays in about $51 \mathrm{~W}, \mathrm{Z}, \mathrm{H}$ quanta. The difference in $\mathrm{QCD}$ is there are no Higgs scalar and its non-zero VEVs, so gluons are massless. This makes the process much more explosive 
because all harmonics with different momenta move together, with the speed of light. As described in [27], we had solved it both numerically and analytically (based on work by Luescher and Schekhter [29]). If one uses notations introduced by Witten (accidentally in the same year, 1977) for spherical YM, it makes it most elegant. 4 components of the potentials can be written as

$$
\begin{array}{r}
\mathcal{A}_{j}^{a}=A(r, t) \Theta_{j}^{a}+B(r, t) \Pi_{j}^{a}+C(r, t) \Sigma_{j}^{a} \\
\mathcal{A}_{0}^{a}=D(r, t) \frac{x^{a}}{r}
\end{array}
$$

with

$$
\Theta_{j}^{a}=\frac{\epsilon_{j a m} x^{m}}{r}, \quad \Pi_{j}^{a}=\delta_{a j}-\frac{x_{a} x_{j}}{r^{2}}, \quad \Sigma_{j}^{a}=\frac{x_{a} x_{j}}{r^{2}}
$$

and functions $A, B, C$, and $D$ can be thought of as ( $r, t$ dependent) Abelian gauge $\left(A_{\mu=0,1}\right)$ and Higgs $(\phi, \alpha)$ field on hyperboloid

$$
A=\frac{1+\phi \sin \alpha}{r}, \quad B=\frac{\phi \cos \alpha}{r}, \quad C=A_{1}, \quad D=A_{0} .
$$

with the action

$$
\begin{array}{r}
S=\frac{1}{4 g^{2}} \int d^{3} x d t\left[\left(\mathcal{B}_{j}^{a}\right)^{2}-\left(\mathcal{E}_{j}^{a}\right)^{2}\right]= \\
4 \pi \int d r d t\left(\left(\partial_{\mu} \phi\right)^{2}+\phi^{2}\left(\partial_{\mu}-a_{\mu}\right)^{2}+\frac{\left(1-\phi^{2}\right)^{2}}{2 r^{2}}\right. \\
\left.\left.-\frac{r^{2}}{2}\left(\partial_{0} A_{1}-\partial_{1} A_{0}\right)^{2}\right)\right)
\end{array}
$$

Omitting all details, let us show directly the promised spherical shell at large times. Those have the following energy density

$$
4 \pi r^{2} e(r, t)=\frac{8 \pi}{g^{2} \rho^{2}}\left(1-\kappa^{2}\right)^{2}\left(\frac{\rho^{2}}{\rho^{2}+(r-t)^{2}}\right)^{3}
$$

Of course, at large times the field becomes weak field which can be decomposed into gluons: the Fourier transform of the fields provides the energy distribution of the resulting gluons. Numerical studies of the problem has been reported in [27] as well. Those are naturally more flexible than analytic and allows for more realistic initial shape of instantons and sphalerons, with exponential (rather than power-like) tails of the fields at large distances ${ }^{6}$.

Alternative derivation of the same explosive solution, using specific conformal mapping from the $\mathrm{d}=4$ spherically symmetric Euclidean solution has been found in [25].

\footnotetext{
${ }^{6}$ The phenomenological reasons for exponential rather than power instanton tail are discussed e.g. in [30].
} 
Now, what about quark pair production? Again, this problem much debated in literature on electroweak theory in 1980's but not yet completely solved. In its general form, the issue is to derive an analog of the index theorem for the case when there are outgoing fields: it suppose to tell us how many fermionic levels have crossed zero (and are produced) based on some topological properties of the gauge field alone. The usual form of it, involving a change in the Chern-Simons number, seem to be obviously incorrect, because its variation is in general (and specifically for the time-dependent solutions we speak about) not an integer.

Further progress in this direction was achieved by derivation of the explicit solution to the Dirac eqn in the background of exploding sphalerons [26]. This solution starts with a static zero energy solution for the YM sphalerons, and then shows how the quarks get accelerated by the electric field into the finiteenergy spectrum of emitted quarks. The energy spectrum of the outgoing quarks have been found to be rather simple

$$
\mathbf{n}_{R}(k)=\frac{4 \pi k^{2}}{2 k}\left|\mathbf{q}_{R}^{\dagger}(\vec{k})\right|^{2}=\rho(2 k \rho)^{2} e^{-2 k \rho} .
$$

The distribution integrates to exactly one produced quark. It is close to Plank spectrum with the effective temperature $T=1 /(2 \rho)$, which is about $300 \mathrm{MeV}$ for a standard $\rho=1 / 3 \mathrm{fm}$. Accidentally, this is close to the initial temperature of quark-gluon plasma in the RHIC energy domain.

This phenomenon leads to production of quarks from 0 up to the whole set of light quark pairs, $\bar{u} u \bar{d} d \bar{s} s$. So tentatively we estimate the yield of partons per cluster to be about 3 gluons and up to 6 quarks+antiquarks.

\section{Initial stage of the High Energy Heavy Ion Collisions}

\subsection{Excitation of the QCD vacuum into the QGP}

We have briefly reviewed recent theoretical literature: now we discuss more phenomenological issues and the numbers involved. Let us start with the question: what is the size $\rho$ and consequently the mass of the QCD topological clusters excited?

The idea of sudden excitation implies that all coordinates of the system are not changed. Size is one of the coordinates, and so it is natural to assume that the cluster size is approximately the same as the mean radii of the instantons populating the QCD vacuum. Using a value $\rho=1 / 3 \mathrm{fm}$ [31], which has passed multiple lattice and phenomenological tests, one obtains the cluster mass of about $3 \mathrm{GeV}$. (For comparison, the mass of electroweak sphalerons is about 10-15 TeV.)

Let me remind some basic facts about the QCD vacuum and instantons. Any quantum-mechanical tunneling lowers the ground state energy, and in QCD it 
is also the case ${ }^{7}$. The instanton contribution to the ground state energy $\epsilon_{\text {vac }}$ is calculated in the models and on the lattice: see [4] for details. A general expression for the non-perturbative shift of the $Q C D$ vacuum energy density is known as the scale anomaly relation ${ }^{8}$

$$
\epsilon_{v a c}=-\frac{b}{128 \pi^{2}}<0\left|\left(g G_{\mu \nu}^{a}\right)^{2}\right| 0>
$$

where $b=11 N_{c} / 3-2 N_{f} / 3$ is the usual coefficient of the beta function of QCD, and the matrix element here is known as the gluon condensate. Each instanton contributes $32 \pi^{2} / g^{2}$ to it, so ${ }^{9}$ the instanton contribution

$$
\epsilon_{\text {vac }}^{\text {instantons }}=-\frac{b}{4} n_{\text {inst }} \approx-(0.5) \mathrm{GeV} / \mathrm{fm}^{3}
$$

where numerical value corresponds to the phenomenological instanton density [31] $n_{\text {inst }}=1 \mathrm{fm}^{-4}$. Note that this energy density [34] is 3 times higher than the weight of the nuclear matter $m_{n} n_{0} \sim .15 \mathrm{GeV} / \mathrm{fm}^{3}$.

One may explain that as follows: we live in a kind of a superconducting phase, and only by colliding heavy ions we can produce a tiny fireball of the "normal" phase, QGP, which has its ground state higher. In order to produce it, we should both (i) create thermally excited quarks and gluons and (ii) "melt" this vacuum energy ${ }^{10}$.

The crucial point we now emphasize is that this amount of energy density is only the lower bound on the actual energy one must spend in order to erase the vacuum fields from QGP. This value would correspond to the adiabatically slow excitation process, in which it would be equal to the work $W=\int p d V$ against the vacuum pressure $p=-\epsilon_{v a c}$. In the high energy collisions we are closer to the opposite limit of instantaneous excitation, so the excitation energy is higher. As a simple example, consider a volume V instantaneously "shocked" so that all instanton sufficiently closed to that moment are "disrupted" and the Yang-Mills field are found with non - integer values of the topological coordinate $N_{C S}$. The lowest energy states in this case is nothing else but the topological potential we are going to study: it is rather expensive. The excitation energy density can be estimated ${ }^{11}$ as that of a gas of "turning states" or clusters $\epsilon \sim M_{\text {clusters }} n_{\text {instantons }} \rho$ where $M_{\text {clusters }} \sim 3 \mathrm{GeV}$ and instanton

\footnotetext{
${ }^{7}$ In the presence of fermions this statement is not generally true. For examples, supersymmetric extensions of both quantum mechanics and QCD have positive shift of the ground state energy, and for QCD with large number of flavors $N_{f}$ it was suggested that the result even oscillates with $N_{f}[32]$.

${ }^{8}$ Although this is often referred to as a "bag term", it is actually 10-20 times the value of the bag constant from the fit of MIT bag model [34].

${ }^{9}$ We have ignored corrections to the action due to instanton interactions and other effects here.

${ }^{10}$ More accurately, at $T=T_{c}$ instantons do not go away but restructure into pairs [4]. They are however get suppressed at $\mathrm{T}$ relevant for RHIC conditions due to Debye-like screening.

${ }^{11}$ All instantons with centers separated in Euclidean time by less than $\rho$ are excited.
} 
size $\rho=1 / 3 \mathrm{fm}$ and density $n_{\text {instantons }}=1 \mathrm{fm}^{-4}$ [31]. With these numerical values, the excitation energy density is about $1 \mathrm{GeV} / \mathrm{fm}^{3}$, a factor 2 higher than in the adiabatic case (20).

Another general point: since the instanton vacuum is rather dilute $n \rho^{4} \sim$ $10^{-2}$ [31], its instantaneous excitation naturally leads to rather dilute system of gluonic clusters (see below).

One more important feature of the QCD vacuum is chiral symmetry breaking, which is also believed to be generated by instantons. Its magnitude is characterized by the quark condensates, which have the following magnitude ${ }^{12}$

$$
<\bar{u} u>=<\bar{d} d>\approx 1.8 \mathrm{fm}^{-3}, \quad<\bar{s} s>\approx 0.8<\bar{u} u>
$$

In total it makes about 5 quark-anti-quark pairs per $\mathrm{fm}^{-3}$, to be compared to about 0.5 valence quarks per $\mathrm{fm}^{-3}$ in nuclear matter. In sudden collisions all condensates should disappear, thus all these vacuum quarks should become real as well.

Let us now have a look at heavy ion collisions. For central AuAu at RHIC energies (which will be our primary example in this paper) the volume occupied by QGP at its maximum is $V_{Q G P} \approx 1000 \mathrm{fm}^{3}$. Considering now the total energy needed to kill instantons (non-adiabatically) is of the order of $1 \mathrm{TeV}$, and total number of quark pairs from eliminated quark condensates is in the thousands. This is comparable to total transverse energy and total hadron multiplicity observed: thus the phenomenon clearly cannot be neglected.

Concluding this subsection, we worn the reader again that the examples given in it are just to get some preliminary orientation with the physics and numbers involved. All of them mentioned came from the instanton liquid model [31] which was designed to explain the mechanism of the chiral symmetry breaking (such as the values of the condensates) and masses of the lowest hadronic states. In this model the only instantons which counts are those sufficiently separated from each other and contributing significantly to the near-zero eigenvalues the quark Dirac operator. In the applications we consider now - high energy collisions - this condition can be lifted. If so, one finds that the total density of instanton-like topological fluctuations in vacuum is actually about one order of magnitude larger.

\subsection{Why heavy ion collisions and not pp?}

Although topological cluster production is argued to be important for understanding of the hadronic cross sections, and in particular their slow growth with energy, the main point of this work is that such phenomena have a chance to be even more important in heavy ion collisions. The reason is related with different cutoff scales in vacuum and QGP.

\footnotetext{
${ }^{12}$ For completeness: unlike the vacuum energy this quantity has non-zero anomalous dimension and thus depends on the normalization scale $\mu$, which is taken to be $1 \mathrm{GeV}$.
} 
In the QCD vacuum the non-perturbative effects generate a "semi-hard" or "substructure scale" $Q^{2} \sim 1-2 \mathrm{GeV}^{2}$, which is both the lower boundary of pQCD as well as the upper boundary of low energy effective approaches (like Nambu-Jona-Lasinio or Chiral Lagrangians).

Quite different pQCD cutoff is however expected for heavy ion collisions. As argued over the years (see e.g. [12]) the final state is in a Quark-Gluon Plasma phase of QCD. It is qualitatively different from the QCD vacuum: there is no confinement or chiral symmetry breaking, and instantons are suppressed as well. Therefore the cut-off in excited matter is expected to be determined by a plasma-like screening: its description in terms of quark and gluon quasi-particles becomes natural. At equilibrium, gluon effective mass is [12]

$$
M_{g}^{2}=\frac{g^{2} T^{2}}{2}\left(\frac{N_{c}}{3}+\frac{N_{f}}{6}\right)
$$

Although the scale in question grows with $\mathrm{T}$, in the window $T=(1-3) T_{c}$ it is actually smaller than the pQCD cutoff in vacuum. Lattice thermodynamics data support it, and fitted quasi-particle masses (see e .g. [36]) are

$$
M_{T}^{g} \approx .4 \mathrm{GeV}, \quad M_{T}^{q} \approx .3 \mathrm{GeV}
$$

Without such low masses one also cannot get high pressure, which is not only seen on the lattice but also in explosive behavior of RHIC collisions.

The schematic picture of scale development with time after collision is shown in Figure 5. A very important consequence of such non-monotonous behavior of the pQCD cutoff implies then, that we can describe gluons (quarks) originating from exploding non-perturbative objects by classical Yang-Mills (Dirac) eqs with better confidence, provided those go into QGP in this window of parameters. This is why heavy ion conditions are so special.

If a topological cluster is produced in hadronic collisions, its expansion is affected at the vacuum scale $\sim 1 \mathrm{GeV}$ itself, by confining forces and other instantons. This is above the mean energy of the spectrum just discussed: it means that it is impossible to use classical YM description in this case.

However if it is produced in AA case and gluons/quarks are emitted as quasi-particles into deconfined QGP, the matter modification only applies to momenta $p<M_{g}, M_{q}$ which is till somewhat smaller than the peak value of the spectrum resulting from the cluster decay. So, although such modifications can be substantial, they cannot destroy most of the classical treatment of the cluster decay.

\subsection{Cluster Production in Heavy Ion Collisions}

We will argue in this section that at RHIC cluster production may contribute significantly to the total amount of entropy produced, and, is probably the leading source of promptly produced quark-antiquark pairs. 


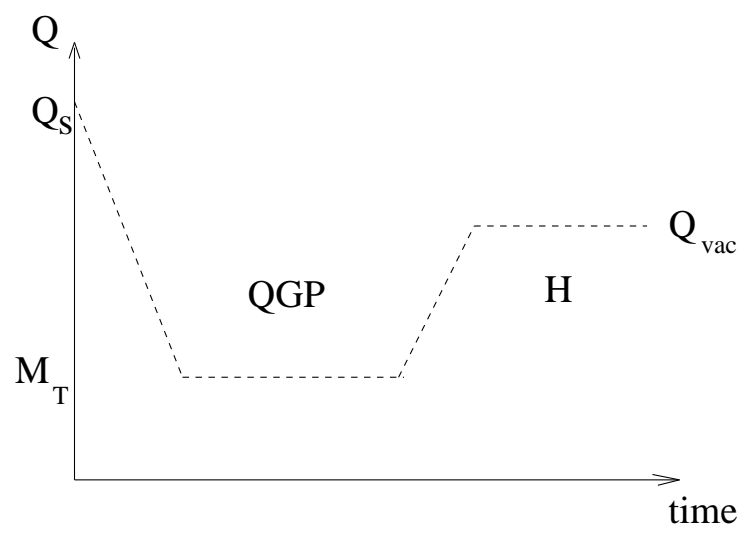

Figure 5: Schematic plot of the cut-off scales during the evolution of the system with time. At the collision time $=0$ the scale is presumably the saturation scale $Q_{s}$ in the incoming nuclei, which grows with the collision energy. Then the cutoff decreases reaching some nearly constant value in QGP, the thermal gluon mass $M_{T}(23)$ and stay at this value till it rises again in the mixed phase to its vacuum value in the hadronic $(\mathrm{H})$ phase $Q_{v a c} \sim 1 \mathrm{GeV}$.

As explained in section 2, the semiclassical ab initio calculation of the sphaleron excitation is not yet available. We will therefore use estimates based on parton-model-style phenomenology of all hadronic collisions, developed by G. W. Carter, D. M. Ostrovsky and myself [30]. The main idea was to identify two components of the hh collisions, the color exchanges and the "color objects production", and deduce the corresponding cross sections at the partonic level. We looked at high energy $N N, \pi N, \gamma N$, and $\gamma \gamma$ cross sections which all increase with energy logarithmically for $\sqrt{s} \sim 100 \mathrm{GeV}$

$$
\sigma_{h h^{\prime}}(s)=\sigma_{h h^{\prime}}\left(s_{0}\right)+X_{h h^{\prime}} \ln \left(s / s_{0}\right)
$$

We identified the two components mentioned above with these two terms, respectively, and concentrated on the last (growing) terms. (In contrast to traditional single-Pomeron fit we do not assume those to be proportional to the first terms.) We studied whether some universal semi-hard parton-parton collisions can explain all known $X_{h h^{\prime}}$. Using fitted structure functions of $N, \pi, \gamma$ and simple scaling - each gluon can be counted as 2 quarks $^{13}$ - we have expressed all of those with only one parameter, the value of the qq cross section. With the

\footnotetext{
${ }^{13}$ Corresponding to $\mathrm{SU}(2)$ Casimir scaling, appropriate for instanton-induced reactions.
} 
fitted value ${ }^{14}$

$$
\sigma_{q q}=1.69 \times 10^{-3} \mathrm{fm}^{2}
$$

we got the rising part of cross sections for 4 hadronic reactions reported in the Table in [30]. They all agree reasonably well with the fits for $X_{h h^{\prime}}$ given by Particle Data Book. In [30] we have also looked at the shadowing corrections, of the second (growing) component by the first in pp, where the cross section growth at any variable impact parameters is known directly from the data. The model qualitatively describe these data as well ${ }^{15}$.

We may now extend our analysis to estimate the number of sphaleron-type clusters produced from excited instantons in heavy ion collisions. For central $A A$ collisions of two nuclei we use first the simplest model, one of two spheres with homogeneously distributed partons. The total parton number is $A N_{q}$, with $N_{q} \approx 12$ being the number of "effective quarks" (quarks number plus twice gluons number) per nucleon ${ }^{16}$.

The total number of $q q$ collisions in this case is easily obtained from the following geometric integral:

$$
\begin{aligned}
N_{\text {coll }} & =8 \pi \sigma_{q q} n_{q}^{2} \int_{0}^{R} d r_{t} r_{t}\left(R^{2}-r_{t}^{2}\right) \\
& =3^{4 / 3} 2^{-5 / 3} \pi \sigma_{q q} n_{q}^{2}\left(\frac{A N_{q}}{\pi n_{q}}\right)^{4 / 3},
\end{aligned}
$$

where the quark density is determined by the nuclear density to be $n_{q}=N_{q} \times$ $0.16 \mathrm{fm}^{-3}$.

With $A=197(\mathrm{Au})$ and the value for the quark-quark cross section given above we got the following upper limit for the production of sphaleron-like clusters at RHIC energies ${ }^{17}$

$$
N_{\text {objects }} \approx 400
$$

(Note that this is the upper limit not because we do not know the growing part of the hh cross sections but simply because the "objects" mentioned here can still be either gluons or colored clusters we discuss, or something else: studies of the cross section alone cannot tell the difference. For that one should seriously study the final state corresponding to high-multiplicity hh events: some ideas what to look at can be found in [10].)

\footnotetext{
${ }^{14}$ Note that simple parametric estimate for this cross section, namely $\pi \rho^{2} n_{\text {inst }} \rho^{4}$ gives the right magnitude.

${ }^{15}$ As in other works, we found that the average index of energy growth, 0.08 or so, is actually much reduced by shadowing, and the true one (seen at large impact parameters) is about twice this value.

${ }^{16}$ Of course, the clustering of partons into "constituent quarks" and nucleons increases the number of collisions, but we will ignore such correlations for now.

${ }^{17}$ We will return to energy dependence of this production later. This estimate is based on effective number of partons integrated from $\mathrm{x}=0.1$ to 1 .
} 
This estimate is also naive in the sense that nuclear structure functions are simply assumed to be A times that of the nucleon. One should correct it for what is known as saturation or nuclear shadowing. So far for gluons this effect is unknown, but hopefully will be clarified soon.

\subsection{The Entropy and Quark production}

When we mentioned above "early QGP production" we meant it in only quite limited sense, namely that it can drive hydro-like collective effects. This implies that that the system is "optically dense" $n \sigma \tau>>1$ so that the regime is collisional, and also that its EOS is close enough to $p / \epsilon=1 / 3$.

Many more fundamental questions can be asked such as: (ii) What is the production history of the total entropy of the system?

(iii) When is the quark part of the QGP produced?

Certainly AA collisions producing thousands of outgoing mesons include thousands of quark-anti-quark pairs produced in the process. However, at AGS/SPS collision energies those are believed to be generated by fragmentation of the QCD strings, or color tubes. This process takes time at least 1-2 fm/c because pair production contains small barrier factor.

At higher energies of RHIC/LHC, it was long believed [12] that the produced highly excited matter remains during its evolution of several $\mathrm{fm} / \mathrm{c}$ mostly a "hot glue", with small quark admixture. Various pQCD calculations all concluded the same [14, 19], as well as those based on classical CGC picture [22].

The basic difference between other kind of glue and topological clusters is that in this case the electric and magnetic fields have a significant collinear component, the invariant $\vec{E} \vec{B}$ is comparable to $E^{2}, B^{2}$. By the chiral anomaly relation, this drives chirality and thus quark production. How it happens in detail during Minkowskian explosion can be seen from the dynamical solution discussed in [26]. It tells us that if the initial zero mode state of the sphalerons is populated, at the end those quarks will get physical (positive) energies $\sim 1 / \rho$.

However, it still remains not quite clear what happens with the fermions during the first (Euclidean) part of the excitation, which will tell us how much that zero level is populated. In the vacuum, virtual quarks are not really bound to a particular instantons but are instead constantly on the way between instantons and anti-instantons. One may think that they equally belong to both ends of the path, and at the moment of sudden excitation there is a stochastic process, and the number of produced quark pairs varies statistically from 0 to $N_{f}=3$.

Assuming for cluster production a naive (UN-shadowed) upper limit discussed above, one gets for central $A u A u$ collisions at RHIC $400 * 3=1200$ gluons and in average about the same number of quarks+antiquarks. Together it is comparable to about a half of the maximal number of partons, limited by the total entropy (observed multiplicity of hadrons) at the end of the collision. The mean energy per parton produced via topological clusters is about 
$3 \mathrm{GeV} / 6 \sim 1 / 2 \mathrm{GeV}$, a relatively small number. If those would equilibrate by themselves, its effective temperature would be only $T \sim 200 \mathrm{MeV}$.

One should compare these numbers with the predictions of other approaches such as CGC model. Based on a numerical solution of the YM equations on transverse lattice [22], at RHIC energies the saturation scale $Q_{s}$ was estimated to be $1.3 \mathrm{GeV}$. The transverse energy per quantum was found to be $1.66 Q_{s}$, resulting in an effective temperature of released gluons $T_{C G C} \approx 1 \mathrm{GeV}$. Thus the gluons from the CGC decay are very hot, hotter than from the topological clusters. The total number of partons or entropy is however roughly comparable.

After the initial formation stage is finished, the CGC and the clusters decayed and QGP is formed, one suppose to obtain the equilibrium initial temperature which is known from hydro calculations. It is about $T_{i} \sim 350 \mathrm{MeV}$, or indeed an intermediate value between the two temperatures mentioned.

\subsection{Collision Energy, Centrality and Rapidity Dependence of Entropy Production}

In fig,1(b) we have shown that both perturbative gluon production via Lipatov vertex and the nonperturbative mechanisms we discuss may originate mostly from a collision of two virtual gluons. As a result, there are similarities between the two processes, and in this subsection we will discuss whether one can use dependence on any of the parameters (mentioned in its title) to separate the two mechanisms. In the first approximation, many features (such as nuclear shadowing etc) are obviously simply common to both of them.

Rapidity distribution of both components is about the same, since both are roughly given by a convolution of two ${ }^{18}$ distribution function ${ }^{19}$. As pointed out by Kharzeev and Levin [37], the interplay of (i) the saturation scale behaving at small $\mathrm{x}$ as

$$
Q_{s}(x) \sim 1 / x^{\lambda}, \quad \lambda=0.25-0.3
$$

with (ii) the saturation conditions itself, leads to characteristic triangular shape of the rapidity distribution ${ }^{20}$

$$
\frac{d}{d y} \sim \exp (-\lambda|y|)
$$

which agrees well with the RHIC data.

Also we expect the centrality dependence of both mechanisms be very similar. Again, following Kharzeev and Levin [37] one can get a very good description

\footnotetext{
${ }^{18}$ We have ignored in both cases possible multi-parton collisions.

${ }^{19}$ The distribution function $\phi\left(x, p_{t}, Q\right)$ depends on parton transverse momentum: its integral over $p_{t}$ is the better known structure function such as $\mathrm{xG}(\mathrm{x}, \mathrm{Q})$. Here $\mathrm{Q}$ Nstands for a normalization scale defining which partons we speak about.

${ }^{20}$ Naturally this expression also agrees with the original parton model of Feynman, which corresponds to $\lambda=0$ and correspondingly a "rapidity-independent plateau".
} 
of it substituting naive number-of-collisions scaling to saturation with its very slow logarithmic dependence $\sim 1 / \alpha\left(Q_{s}(b)\right)$ of density of common small-x gluons produced by a row $\left(\sim A^{1 / 3}\right)$ of nucleons. It is not surprising, since it is a feature of the wave function of colliding nuclei itself.

The dependence on the collision energy however is expected to be quite different. The usual parton model, involving convolution of two parton densities with the power law behavior of the structure function $x G(x) \sim 1 / x^{\lambda}$ would predict also a power growth of the number of partons produced

$$
N(s) \sim s^{\lambda}
$$

However, the saturation scenario reduces this power to a twice smaller power, $\lambda / 2$, see [37]. The reason for that is the energy dependence of the saturation scale itself, 28.

So far we have implicitly assumed the cluster production scale being fixed by cluster mass, or the instanton size in the QCD vacuum, which is some sindependent fixed scale. We also implied that heavy ion collisions at RHIC have $Q_{s} \approx 1 \mathrm{GeV}$, which is the same as the semi-hard instanton scale. If so, the only difference between a single gluon Lipatov vertex and the instanton-induced cluster production is just a factor in the vertex.

However if we consider energy dependence and ask what happens at much higher energies, such as LHC ones and beyond, one should have a better look at scales involved. We will only provide an estimate, assuming the saturation scenario, with $Q_{s}$ increasing as a small power of s. Simple one gluon production, described by the Lipatov vertex $V_{L} \sim \alpha_{s}\left(Q_{s}\right)$ decreases with energy logarithmically. If one naively substitutes the saturation scale into the instanton-induced amplitude

$$
V_{\text {inst }} \sim \exp (-S) S^{2 N_{c}} S=2 \pi / \alpha_{s}\left(Q_{s}\right)
$$

one finds a power-like decrease ${ }^{21}$. It implies [10] that at energies high enough so that the saturations scale would be significantly larger than the ordinary instanton scale, one should expect suppression of cluster production.

This conclusion is naive for at least two reasons:

(i) Although it is in agreement with a general trend of instanton suppression in high field, high temperature and high density [34], one has to look at each issue individually. It was shown by [35] that dense saturated CGC of a single nuclei does not actually suppress instantons ${ }^{22}$ : this can only happen in space-time region after the collision. On the other hand, "killed vacuum instantons" will always contribute, but their number is limited and does not grow with energy. (ii) The saturation scale is not the scale of transverse momenta/virtualities

\footnotetext{
${ }^{21}$ The contribution of "interrupted vacuum instantons" always remains, but would be negligible at very high energies, as it can only provide constant contribution to multiplicity.

${ }^{22}$ They did it by an explicit calculation: going into the rest frame of the nuclei can convince the reader much easier.
} 
which all the partons have. By its definition, it is only a place where the distribution over them changes, from a dilute to a saturated regime. Thus a convolution over all momentum scales with realistic instanton size distribution has to be evaluated, to establish accurate energy dependence of the instantoninduced processes. Such a calculation is not done yet.

\subsection{Heavy Quarkonia Suppression}

In this subsection we consider one more possible signature of cluster explosion: strong $J / \psi$ "ionization" by the dipole excitation similar to those responsible for photo-effect of ordinary atoms. The effect in QGP context has been noticed already in the very first paper discussing possible QGP signals [12]. Later Matsui and Satz [38] noticed that due to a Debye screening in QGP $J / \psi$ and other excited states of $\bar{c} c$ cannot even exist inside QGP as a bound states. Since that time, multiple literature on the subject discusses theory of $J / \psi$ suppression and its relation with experimental data from CERN NA38/50 experiment. RHIC data are expected soon, and prediction range from near total suppression to even $J / \psi$ enhancement, due to possible recombination of two charm quarks at the hadronization stage.

Without going into detailed discussion of current situation, we just evaluate the probability to dissociate different quarkonia states by the gluo-electric field of expanding shells resulting from the cluster explosion.

The cross section for a gluon (with momentum $\mathrm{k}$ ) to dissociate a quarkonium state, denoted as $\Phi$ is [39]

$$
\sigma_{g \Phi}=\frac{2 \pi}{3}\left(\frac{32}{3}\right)^{2}\left(\frac{M}{\epsilon}\right)^{1 / 2} \frac{(k / \epsilon-1)^{3 / 2}}{M^{2}(k / \epsilon)^{5}}
$$

where $\Phi$ is assumed to be a Coulomb system made of quarks with mass $\mathrm{M}$ and binding energy $\epsilon$.

Since the wall of the exploding cluster is a coherent field, in principle one should go back to the original calculation and calculate matrix element of excitation using time-dependent perturbation theory and appropriate time shape of the pulse. However, since the maxima of the gluon spectra from cluster decay and the cross section for the $J / \psi$ dissociation given above happen to be nearly coincident, one can simply estimate it using the incoherent cross section enhanced by a coherence factor ${ }^{23} N_{g}=4-5$. (The fields of all gluons are the same and add in a field strength, not in intensity.)

If the quarkonium is at distance $\mathrm{R}$ from the original position of the cluster, the probability to be dissociated is

$$
P=N_{g} \sigma_{g \Phi} \frac{N_{g}}{4 \pi R^{2}}
$$

${ }^{23}$ The reader should not confuse this factor with a number of outgoing partons at the end. One may say that at the beginning the cluster is made of $N_{g} \sim 6$ gluons, and at the end of 3 gluons plus $0-3 \bar{q} q$. 
where the unusual extra factor $N_{g}$ is the coherent enhancement. Averaging it over the volume, assuming the space is divided into spherical cells of radius $R_{c}$ around each cluster, we get

$$
P=N_{g}^{2} n_{c} \sigma_{g \Phi} R_{c}
$$

where $n_{c}=1 /\left(4 \pi R_{c}^{3} / 3\right)$ is the cluster density.

One can see that for expected $n_{\text {clusters }} \sim 1 \mathrm{fm}^{-3}$ one gets $\mathrm{P}_{J / \psi}>1$, which means that nearly all $J / \psi$ are expected to be dissociated.

The situation is different for $\Upsilon$ and its relatives. First of all, the maximum of the cross section is down by about an order of magnitude. Second, the overlap between the gluon spectrum and the cross section (32) is small: exact numbers also depend on (relatively uncertain) high energy tail $k>1 \mathrm{GeV}$ of this spectrum. As a result, an effective cross section is only $\sim 0.1 \mathrm{mb}$ and the probability of Upsilon excitation is just few percents.

Presumably remnants of "classical glue", which according to calculations in [22] have higher effective temperature $T_{\text {eff }} \sim 1 \mathrm{GeV}$ are more effective in Upsilon dissociation.

\section{Jet Quenching and the explosive edge}

Jet quenching is a kind of "tomography" of the excited system, created in high energy heavy ion collisions. It is based on the fact that even large- $p_{t}$ jets are partially absorbed ${ }^{24}$ during their passage through the system. Jet quenching is thus a tools allowing us to get information about very early stages of such conditions. The so called quenching factor $Q\left(p_{t}\right)$ is defined as the observed number of high- $p_{t}$ hadrons in AA collisions divided by the expected number calculated in the parton model, scaled from pp by a number of collisions. For pions this factor is found to be at RHIC $Q\left(p_{t}\right) \sim .2, p_{t}>4 \mathrm{GeV}$, smaller than expected from pQCD estimate. What role in in is played bu the initial state interaction - the so called saturation or nuclear shadowing of structure functions, and/or Cronin effects - should be clarified in the ongoing deuteron-Au run.

Let us now turn to the theory of jet quenching. In early papers on the subject [40] only parton scattering in Quark-Gluon Plasma (QGP) were discussed. Account for radiation losses [41] and Landau-Pomeranchuck-Migdal (LPM) effect [42] have finalized expectations based on the picture of independent scattering centers.

In recent work [43] a new picture has been proposed, that instead of incoherent scattering points the matter is actually made of coherent classical field, CGC or topological clusters. The first generic enhancement of the radiation is due to coherence effect of classical glue: the field strength are added, not

\footnotetext{
${ }^{24}$ Or deformed: see discussion below on experimental strategies.
} 
intensities, of all gluons making the expanding wall. (This would also work for classical glue created by a materialized CGC.)

The second specific enhancement is due to the fact that expanding thin wall geometry maximizes both the field strength (compare to CGC gluons which occupy all the space equally) and the probability to collide with it. The last point may be especially important: it is hard for a jet to avoid the foam-like structure of expanding shells.

We will not of course go into a rather involved calculations of synchrotron-like radiation in a constant gluo-magnetic field performed in [43], and just mention its main result for the quark energy loss

$$
\frac{\Delta E}{E} \sim 0.21\left(\frac{H}{0.2 \mathrm{GeV}^{2}}\right)^{2 / 3}\left(\frac{1 \mathrm{GeV}}{E}\right)^{1 / 3} .
$$

where $H$ is the field in the shell. The gluon loss scales with the pertinent color Casimir and is about twice larger. Convoluting such losses with the spectrum observed one finds quenching factors of about one order of magnitude, which is in the ballpark of observations.

Let us further note that two mechanisms of radiative energy losses discussed above, namely (i) due to multiple uncorrelated scattering with small momentum transfer, and (ii) one (or few) stronger scatterings can in principle be separated experimentally. The difference between final states become apparent when one asks a question: where the radiated energy go? Theoretically, when the emitting parton and its radiation are separated (become incoherent), the radiation process is considered completed. Experimentally however we cannot know anything about coherence and just look at secondary hadrons, either individually or calorimetrically. In the former case (i) most of the "radiated" energy still is in forward narrow cone. One may even view this mechanism of jet quenching as a "matter modification of a fragmentation function", with a conserved total energy and re-distributed hadron momenta. In the latter case (ii) (which we propose) the situation is different. Radiated gluons are scattered by a strong field to large angels. Therefore, their energy is not in the narrow cone along the jet and it cannot be recovered. This observation may give us a chance to understand which mechanism is responsible for jet quenching.

Summarizing this section: the magnitude of the jet quenching may be explained, by either CGC or cluster scenario. However, as we have already indicated in the list of "RHIC puzzles", the most surprising observations are rather two other features: large azimuthal asymmetry and unusual baryon/meson ratio at $p_{t}>2 \mathrm{GeV}$.

Let me add a bit to discussion of $v_{2}$ at large $p_{t}$. It was found in my work [44] that very strong jet quenching leads to some well-defined "geometric limit" of $v_{2}$ corresponding to the surface emission, but even this value falls below the experimental data. This original conclusion, reflected even in the title of 
this paper. However by the time the paper's proofs were made, STAR have performed additional studies of the 4-body cumulants [45] and concluded that about 15 percent of the original $v_{2}$ was due to non-flow 2-body correlations. That brought the data into agreement with the geometric limit for the sharpedge nuclei. Further account for a "nuclear skin" [46] have however lowered the geometric limit as well, and so the contradiction between data and the geometrical limit is still there.

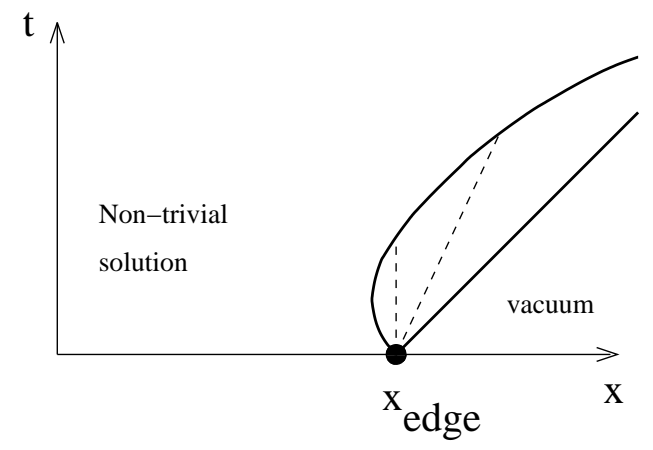

Figure 6: Schematic location of the Riemann wave solution, on the plane the time $\mathrm{t}$ - the space $\mathrm{x}$, between the nontrivial solution of the hydro equations and empty space. Two solid line separating it are the light cone at the right and deflagration front on the left. Rapidity and thermodynamical quantities are constant on straight lines originating from the edge, shown by the dashed lines.

\subsection{The Explosive Edge: hydro treatment}

Unusual phenomena at large $p_{t}$ observed at RHIC are clearly related with the surface of the excited system produced in the collision. This is clear already from the significant quenching of spectra, effectively excluding the central region, but also from large azimuthal asymmetry, and absence of the backward jets in events with high- $p_{t}$ trigger. Furthermore, the data on $v_{2}\left(p_{t}\right)$ show that hydro-generated behavior at $p_{t}<2 \mathrm{GeV}$ joins very smoothly to a new (so far unexplained) regime at $p_{t}>2 \mathrm{GeV}$. Also, it was observed that $v_{2}$ for baryons is somewhat larger than for mesons: natural for hydro regime it seem to be true at higher $p_{t}=(2-6) \mathrm{GeV}$ as well. All this hints to a possibility that the basic physics in the hydro domain and that at higher $p_{t}$ are somewhat related.

There is however a generic problem, preventing us from combining hydro and jet physics into any combined model: their quite different time scales. Indeed, jets move with a speed of light, and cannot be emitted too far from the 
surface: so escaping jet fragments can only interact with matter for a short time, $t_{\text {quenching }} \sim 1 \mathrm{fm} / \mathrm{c}$. Hydro flow, on the other hand, need longer time to be developed, about $t_{\text {hydro }} \sim 10 \mathrm{fm} / \mathrm{c}$ for radial and about half of that for elliptic flow.

The way out of this dilemma proposed in this section is to focus on the specific collective phenomena which may develop during the short time $t_{\text {quenching }}$ at the edge of the fireball. We will do so first in the hydrodynamical context, where the analytic answers can be obtained. This however can only be used as qualitative indications since the size of the system (or the particle number) is not large enough to justify this approach quantitatively, In the second subsection we suggest that multiple explosion of clusters may also "jump-start" collective phenomena at the surface.

Let us start with the generic problem, of an explosion of a system which has a sharp edge. The main point to make here on the onset is that phenomena near the edge basically drive explosions in $1 \mathrm{~d}$, which are much more robust that those in $2 \mathrm{~d}$ and $3 \mathrm{~d}$.

Do we have an edge in heavy ion collisions? Indeed, if one ignores "skin" of nuclei and treat them as drops of nuclear matter with a sharp spherical surface, the almond-shaped overlap region of two nuclei colliding with the impact parameter $b$ will have a sharp edge at which the energy (also particle and entropy) density vanishes as $\epsilon(x) \sim \sqrt{x-x_{e d g e}}$. From hydrodynamical equation it then follows that the acceleration at this point is formally infinite ${ }^{25}$

$$
\frac{\partial v}{\partial t} \approx \frac{1}{\epsilon+p} \frac{\partial p}{\partial x} \sim \frac{1}{x-x_{\text {edge }}}
$$

It is well known how the problem is resolved, at least mathematically, in the framework of hydrodynamical equations. The singularities in the initial conditions are extensively discussed in textbooks such as [47]: let me remind the main idea only. Although hydro admits discontinuities (such as shocks), they always come with certain conditions on them (such as Gugonio/Taub adiabats etc) which the initial conditions in general do not obey. Therefore, a generic situation is that a singular point in the initial conditions opens up into a whole region of special solution, separated by 2 discontinuities from other regions.

A singularity of the type we discuss opens into a region filled with the so called Riemann wave in which rapidity and thermodynamical quantities can be viewed as functions of each other. The region of this solution is schematically shown in Fig.6, it is separated from vacuum by a light cone $t=x-x_{\text {edge }}$ at which matter content vanishes, and from the non-trivial hydro solution on the left by a deflagration front. (The latter curves at later time because the developed hydro rapidity reaches values larger than that of the deflagration.)

\footnotetext{
${ }^{25}$ If the edge is regulated by the usual skin with a Fermi-type distribution, the acceleration is finite and constant at large $\mathrm{x}$, but inversely proportional to small skin width.
} 
The Riemann wave solution itself in relativistic flow is discussed in [47], problem 1 to section 134. It can be summarized by two equations

$$
\begin{gathered}
y=\int \frac{c_{s} d \epsilon}{p+\epsilon} \\
x-x_{\text {edge }}=t * \tanh \left(y-y_{s}\right)
\end{gathered}
$$

where $y, y_{s}$ is matter and sound rapidity, $v=\tanh (y), c_{s}=\tanh \left(y_{s}\right) . \quad \epsilon$ is energy density, $p$ is pressure and $c_{s}^{2}=d p / d \epsilon$ is squared sound velocity. If $p, \epsilon$ is a function of one variable (e.g. temperature) the meaning of the integral in the former equation is clear. If there are more conserved quantities and chemical potentials, it should be taken along the adiabatic path in the phase diagram the systems takes while cooling down. In particular, for QGP or resonance gas with a simple $\operatorname{EoS} c_{s}^{2}=$ const this first equation establishes a very simple direct relation between particle/entropy density $\sim T^{1 / c_{s}^{2}}$ and rapidity, namely

$$
\frac{n}{n_{0}}=\exp \left(-y / c_{s}\right)
$$

each of which holds at the whole line originating from the edge. The light cone corresponds to $y \rightarrow \infty, n \rightarrow 0, n_{0}$ is the density at rapidity zero, etc. Of course, exponent in rapidity is a power law in momenta/energies, and its index is rather unusual. For QGP Eos it gives $1 / p^{\sqrt{3}}$, with a somewhat larger power 2-3 if one uses more appropriate EoS of the resonance gas. However in all cases the spectrum generated by the Riemann wave is much harder than the observed particle spectrum, which has larger effective power of about 10-12.

Let us now discuss limitations on hydrodynamics stemming from the fact that the particle multiplicity in our problem is not like $10^{23}$ as in air/water but rather limited. At central AuAu collisions at RHIC at rapidity $y \sim 0$ one actually finds ${ }^{26}$

$$
\frac{d N_{\text {hadrons }}}{d \eta d \phi} \sim \frac{d N_{\text {partons }}}{d \eta d \phi} \sim 150
$$

where $\eta, \phi$ are pseudo-rapidity and azimuthal angle. This means that when we go about 2 orders of magnitude down the spectra we reach of only about 1 particle per unit solid angle, and should stop using hydrodynamics. More elaborate estimates using viscosity corrections [48] lead to the same conclusion.

This simple idea put a cutoff of hydro to $p_{t} \sim 1.7-2 \mathrm{GeV}$, which is indeed where deviations from hydro predictions are observed. It also indicate to us that the above discussion of the Riemann wave can only be used as a qualitative indication, and its comparison to data is not really justified.

\footnotetext{
${ }^{26}$ We mean all kinds of hadrons together, charged and neutral. The partons are not directly observed, of course, but believed to be about the same to justify the approximately adiabatic expansion which works well in hydro applications and confirmed by cascades.
} 


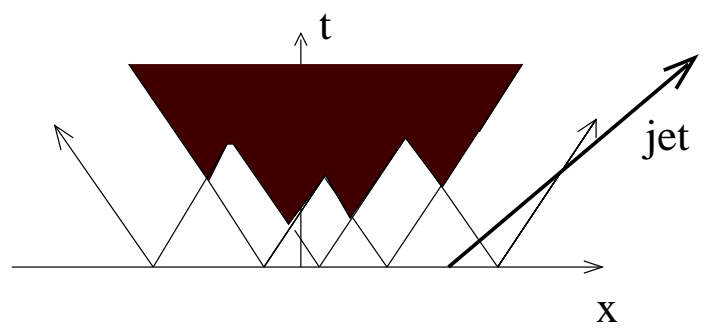

Figure 7: Schematic picture of initial stage of the heavy ion collision, on the plane transverse coordinate $\mathrm{x}$ - time $\mathrm{t}$. At $\mathrm{t}=0$ several clusters are produced which promptly explode into empty spherical shells, shown by two divergent lines. The decoherent quarks and gluons - QGP shown as the shaded regionis produced in secondary collisions of these shells, but partons at the edge fly away.

\subsection{Exploding Clusters at the edge}

We have concluded at the end of the previous section, that hydro can provide only qualitative ideas about matter evolution at the edge of the system, and thus some microscopic approach is needed.

This is of course very much limited by our poor knowledge of the matter conditions at the early time. However the generic idea - that particles at the edge of the system experience collisions with a stream of particles from one side only and are therefore accelerated more rapidly than any others - holds in one form or another for any models under consideration.

In a perturbative mini-jet scenario with a modest re-scattering one simply would find a fraction of outward moving mini-jets which never re-scattered, but fragment into secondary hadrons independently. Naturally, those would spatially separate from those which did re-scatter, and create thin collisionless "atmosphere" receding from the fireball with (transverse) rapidity $y_{t} \sim 1$.

In a CGC picture one should study the behavior of the classical Yang-Mills equations which starts with the initial conditions having a sharp edge. Although it is not yet done, by analogy to discussion of hydro equation in the preceding section, one may expect the edge also to act as a focal plane of an explosive expansion into vacuum. Moreover, in terms of effective pressure the non-linear term in YM equation is even stronger than in the EoS of QGP, so more dramatic phenomena can be expected.

Finally, in the scenario advocated in this paper, namely on multiple production of the topological clusters, one get a picture shown schematically in Figure 7. The message displayed there is very simple: although most expanding spherical shells from the cluster explosion collide and produce QGP, as discussed above, the part of those shells going from the edge toward the empty space cannot be not stopped in this way. 
So, if in all pictures of the initial stage one finds partons receding from the system edge outward, it is obviously interesting to know what happens with them? The answer to this question depends on their density. In pp collisions, with a couple of mini-jets or a single cluster produced, confining flux tubes would slow down their propagation and drain their energy into flux tubes fragmentation (hadronization a la Lund model).

We would argue that the situation should be different if the outward moving partons are sufficiently numerous to neutralize each other in color and thus fly away without strings attached. In other words, we suggest that parton fragmentation in AA collisions is different from independent one observed in $e+e-$ and $p p$ collisions.

We will further suggest that even fragmentation of high- $p_{t}$ jets should be affected, as they fly through the comoving matter originated from the explosive edge, see Figure 7. A number of detailed models of such kind are under numerical investigation now, with results to be reported elsewhere [46]. Hopefully those provide some insight into the puzzles of large $v_{2}$ and high baryon content.

\section{Event-by-event Fluctuations}

First, a brief history. This approach was first proposed [49] as a tool to test statistical methods and extract more accurate data about freeze-out. More radical ideas of its applications included a search for the QCD tri-critical point [50] and even for quark-gluon plasma [51].

Experimentally, it has been pioneered by NA49 experiment at CERN [52] which found that non-statistical event-by-event fluctuations in mean $p_{t}$ are basically absent, if measurements are made for rather forward rapidities. Late data by this group, as well as by CERES experiment, have found dynamical effect at mid-rapidity roughly consistent with standard resonance production: no trace so far neither of the tri-critical point nor of survived QGP fluctuations.

The first STAR preliminary data on mean $p_{t}$ fluctuations have been discussed in a number of meetings by T.Trainor and G.Roland, but unfortunately no publications are so far available.

Cluster production induce event-by-event fluctuations because their number is significantly smaller than the number of particles. The estimates thus follows the ordinary statistical arguments, assuming separate cluster production is independent. The original presentation of this idea has been made at the 2001 CERN workshop [53]. Discussion of the elliptic flow or $v_{2}$ fluctuations of the same origin is discussed in a separate paper [54].

The relative fluctuation in number of clusters is

$$
\frac{\delta d N_{\text {clust }} / d y}{d N_{\text {clust }} / d y} \sim\left(\frac{1}{d N_{\text {clust }} / d y}\right)^{1 / 2}
$$

For central AuAu collisions at RHIC this fluctuation is about .1. Assuming the 
fraction of in total parton multiplicity coming from clusters, $f_{\text {clust }} \approx 1 / 2$, and that the other component is fluctuating much less, we then expect the observed particle density per unit rapidity $d N / d y$ at mid-rapidity of RHIC AuAu central collisions to fluctuate by about by 0.05 . (This of course can be directly tested experimentally).

The next step is to estimate the expected fluctuations in hydro expansion velocity:

$$
\frac{\delta v_{t}}{v_{t}}=\frac{\delta d N / d y}{d N / d y} \frac{\partial \log \left(v_{t}\right)}{\partial \log (d N / d y)}
$$

The log-log derivative, also called index, depends on the EOS and can be calculated from hydrodynamics. Simple estimate follows from a comparison of mean flow at SPS and RHIC (or at two RHIC energies). At $\sqrt{s}$ changing from 17 to $130 \mathrm{GeV}$ the charge in hadron multiplicity $d N / d y$ is about 1.5 while the flow velocity changed only from .55 to about .65: thus the log-log derivative is about .4 , so relative fluctuation in the velocity is estimated to be about 0.02 .

The so called $m_{t}$ slopes, or effective temperatures $T_{\text {slope }}$, contains $v_{T}$ via the so called blue shift factor

$$
T_{\text {slope }}=T_{\text {true }} \sqrt{\frac{1+v_{t}}{1-v_{t}}}
$$

Differentiating this over $v_{t}$ we finally get the estimate for the "temperature fluctuation" at RHIC relative to SPS (where cluster production is assumed to be negligible)

$$
\frac{\delta T_{\text {slope }}}{T_{\text {slope }}}=\frac{\delta v_{t}}{v_{t}} \frac{v_{t}}{1-v_{t}^{2}}
$$

or about 0.01 in central AuAu collisions. A relative increase in fluctuation in STAR relative to NA49 is indeed of the magnitude corresponding to about 1 per cent "slope fluctuations" for the central heavy ion collisions.

Another way to estimate the log-log derivative or index is directly from the hydrodynamical calculations.. Fig.14 of the second ref. of [13] show dependence of $\left\langle m_{t}\right\rangle-m$ for pions and nucleons on the input multiplicity $d N / d y$. Using these results for two highest values, $d N / d y=466,669$, we get that the corresponding log-log derivative

$$
P_{h}=\frac{\partial \log T_{\text {slope }}}{\partial \log d N / d y}
$$

are $P_{\pi}=.37$ for pions and $P_{N}=.63$ for nucleons. Together with

$$
\frac{\delta T_{\text {slope }}}{T_{\text {slope }}}=P_{h} \frac{\delta d N / d y}{d N / d y}
$$


The resulting prediction is again about 1 percent fluctuation of slope for pions but about 2 percent for nucleons. (This difference is natural since nucleons are more sensitive to flow in general.)

More direct tests of whether event-by-event fluctuations of mean $p_{t}$ are indeed due to the $p_{t}$-slope fluctuations, or something else, can be done experimentally using complete two-body correlation function. Simple summary of the idea can be expressed as follows: Slope fluctuation produce positivecorrelation when two transverse momenta are either both small or both large, but a negative one if one momentum is large and one small. Preliminary indications are that it is indeed the case: we are waiting for data to get finalized and public, to test it further.

Finally, a comment on other possible kind of event-by-event fluctuations, those related with the non-zero topology of the clusters, which (again with relative probability $\sim 1 / \sqrt{N_{\text {clusters }}}$ which is large compared to statistical fluctuations $\sim 1 / \sqrt{N_{\text {particles }}}$ ) would produce an observable dis-balance of leftversus-right quarks. Like in electroweak theory in Big Bang before electroweak transition, we think that thermal rate in QGP should be big enough to erase this effect.

\section{Summary and Discussion}

We have discussed in this work multiple consequences of the idea, that quantum mechanical excitation from under the QCD topological barrier can be an important component of high energy heavy ion collisions, and at RHIC energies $(\sqrt{s} \sim$ hundreds of $\mathrm{GeV})$ in particular. We thus proposed that more traditional views of the initial stage of such collisions be supplemented by a "firework" of mini-explosions of topological clusters, the remnants of vacuum instantons. Both their shape and further explosive behavior are determined from classical YM and is under control, numerically and analytically. We pointed out that the low scale of the thermal masses can be used to justify application of classical Yang-Mills equations, as clusters explodes into QGP. Each cluster leads to production of about 3 gluons $+(0-3) \bar{q} q$. Prompt production of quarks by this mechanism is especially unique, and may leads toward QGP, although not quite in the equilibrium $\mathrm{g} / \mathrm{q}$ ratios.

We made rough estimates of the number of clusters produced based on parton-parton cross section obtained phenomenologically (more work on its direct calculation is still needed) and found its upper limit to be comparable to amount of entropy actually produced. Other mechanism (such as Color Glass Condensate advocated by McLerran-Venugopalan model) also may produce a similar entropy but with much higher temperature. A combined entropy and energy of both components roughly correspond to initial properties of equilibrated QGP, known from hydro applications.

The signature of the topological clusters is thus prompt production of quark- 
anti-quark pairs. With lepton and/or photons from early stages to be eventually observed, one would be eventually be able to probe the quark/gluon ratio at early stages experimentally. Another place where prompt quark production is needed is to explain large baryonic component at high $p_{t}$. Although we have not proposed a specific model for it, we argued that the existence of the "explosive edge" containing quark pairs is likely to be related with their origin.

We further argued that cluster production is expected to become sub-leading at energies much higher than RHIC, at LHC, provided the saturation scale there is indeed increased well above the instanton scale.

We further speculated that cluster production can help us with some other RHIC puzzles, such as enhanced event-by-event fluctuations and jet quenching. These estimates are to be compared with RHIC data, as they will become more detailed and accurate.

Acknowledgments Let me thank my collaborators G.Carter,J.Casalderry, R.Janik, D.Ostrovsky and I. Zahed: this paper is in many ways a summary of ideas which have been developed in the course of joint work with them. I also would like to thank D.Kharzeev,L.McLerran and R.Venugopalan for useful discussion of their papers. This work is partially supported by the US-DOE grant No. DE-FG02-88ER40388. The main part of it has been written in ITP, Santa Barbara provided support under NSF Grant PHY99-07949. Kind hospitality and very interesting discussions with people in ITP and especially with David Gross is acknowledged.

\section{References}

[1] E. Shuryak, arXiv:hep-ph/0205031. Also plenary talk at Quark matter 02 of the same title, to be publihed as proceedings in Nucl.Phys.A.

[2] A. A. Belavin, A. M. Polyakov, A. S. Shvarts and Y. S. Tyupkin, Phys. Lett. B 59, 85 (1975).

[3] G.t'Hooft Phys. Rev. D 14, 3432 (1976) [Erratum-ibid. D 18, 2199 (1976)].

[4] T. Schafer and E. V. Shuryak, Rev. Mod. Phys. 70, 323 (1998) [arXiv:hep$\mathrm{ph} / 9610451]$.

[5] A.Ringwald, Nucl.Phys. B330 (1990) 1, O.Espinosa, Nucl.Phys. B343 (1990) 310; V.V. Khoze, A. Ringwald, Phys.Lett. B259:106-112, 1991 V.I.Zakharov, Nucl.Phys. B353 (1991) 683: M.Maggiore and M.Shifman, Phys.Rev.D46:3550-3564,1992

[6] D. Diakonov and V. Petrov, Phys. Rev. D 50, 266 (1994) [arXiv:hep$\mathrm{ph} / 9307356]$.

[7] A. Ringwald and F. Schrempp, Phys. Lett. B 503, 331 (2001) [arXiv:hepph/0012241]. arXiv:hep-ph/0006215. F. Schrempp, arXiv:hep-ph/0109032. 
[8] D. E. Kharzeev, Y. V. Kovchegov and E. Levin, Nucl. Phys. A 690, 621 (2001) [arXiv:hep-ph/0007182].

[9] M. A. Nowak, E. V. Shuryak and I. Zahed, Phys. Rev. D 64, 034008 (2001) [hep-ph/0012232].

[10] E. V. Shuryak, Phys. Lett. B 515, 359 (2001) [hep-ph/0101269].

[11] P.Faccini, Talk at Yale workshop on resonance production, Dec.2002.

[12] E. V. Shuryak, Phys. Lett. B 78, 150 (1978) Sov. J. Nucl. Phys. 28, 408.1978 YAFIA,28,796 (1978). Phys. Rept. 61, 71 (1980).

[13] D. Teaney, J. Lauret and E. V. Shuryak, Phys. Rev. Lett. 86, 4783 (2001) [arXiv:nucl-th/0011058], arXiv:nucl-th/0110037. P. F. Kolb, P. Huovinen, U. W. Heinz and H. Heiselberg, Phys. Lett. B 500, 232 (2001) [arXiv:hepph/0012137]. P. Huovinen, P. F. Kolb, U. W. Heinz, P. V. Ruuskanen and S. A. Voloshin, Phys. Lett. B 503, 58 (2001) [arXiv:hep-ph/0101136].

[14] K. Geiger and B. Muller, Nucl. Phys. A 544, 467C (1992).

[15] L. Xiong and E. Shuryak, Nucl. Phys. A590, 589C (1995).

[16] D. Molnar and M. Gyulassy, Nucl. Phys. A 697, 495 (2002) [nuclth/0104073].

[17] P.Arnold, G.D.Moore, L.G.Yaffe JHEP 0011 (2000) 001

[18] G. Policastro, D. T. Son and A. O. Starinets, Phys. Rev. Lett. 87, 081601 (2001) [hep-th/0104066].

[19] R. Baier, A. H. Mueller, D. Schiff and D. T. Son, Phys. Lett. B 502, 51 (2001) [arXiv:hep-ph/0009237]. arXiv:hep-ph/0204211.

[20] L. V. Gribov, E. M. Levin and M. G. Ryskin, Phys. Rept. 100, 1 (1983). J. P. Blaizot and A. H. Mueller, Nucl. Phys. B 289, 847 (1987).

[21] L. D. McLerran and R. Venugopalan, Phys. Rev. D 49, 2233 (1994) [arXiv:hep-ph/9309289].

[22] A. Krasnitz, Y. Nara and R. Venugopalan, Phys. Rev. Lett. 87, 192302 (2001) [arXiv:hep-ph/0108092].

[23] N.Manton, Phys.Rev.D 28 (1983) 2019; F.R.Klinkhamer and N.Manton, Phys.Rev.D30 (1984) 2212.

[24] V. A. Rubakov and D. T. Son, Nucl. Phys. B 424, 55 (1994) [arXiv:hep$\mathrm{ph} / 9401257]$. 
[25] R. A. Janik, E. Shuryak and I. Zahed, "Prompt multi-gluon production in high energy collisions from singular Yang-Mills solutions," arXiv:hepph/0206005. Phys.Rev.D, in press.

[26] E. Shuryak and I. Zahed, "Prompt quark production by exploding sphalerons," arXiv:hep-ph/0206022. Phys.Rev.D, in press.

[27] D. M. Ostrovsky, G. W. Carter and E. V. Shuryak,Phys. Rev. D 66, 036004 (2002) arXiv:hep-ph/0204224.

[28] J. Zadrozny, Phys. Lett. B 284, 88 (1992). M. Hellmund and J. Kripfganz, Nucl. Phys. B 373, 749 (1992).

[29] M.Luscher, Phys.Lett.B70 (1977) 321; B.Schechter, Phys.Rev.D16 (1977) 3015.

[30] G. W. Carter, D. M. Ostrovsky and E. V. Shuryak, Phys. Rev. D 65, 074034 (2002) [arXiv:hep-ph/0112036].

[31] E. V. Shuryak, Nucl. Phys. B 203, 93 (1982).

[32] M. Velkovsky and E. V. Shuryak, Phys. Lett. B 437, 398 (1998) [arXiv:hep$\mathrm{ph} / 9703345]$.

[33] E. Kuraev, L. Lipatov and V. Fadin, Sov. Phys. JETP 45 (1977) 199; I. Balitsky and L. Lipatov, Sov. J. Nucl. Phys. 28 (1978) 822; L. Lipatov, Sov. Phys. JETP 63 (1986) 904.

[34] E. V. Shuryak, Phys. Lett. B 79, 135 (1978).

[35] D. E. Kharzeev, Y. V. Kovchegov and E. Levin, Nucl. Phys. A 699, 745 (2002) [arXiv:hep-ph/0106248].

[36] P. Levai and U. W. Heinz, Phys. Rev. C 57, 1879 (1998) [arXiv:hep$\mathrm{ph} / 9710463]$.

[37] D. Kharzeev and E. Levin, Phys. Lett. B 523, 79 (2001) [arXiv:nuclth/0108006]. D. Kharzeev, E. Levin and M. Nardi, arXiv:hep-ph/0111315.

[38] T. Matsui and H. Satz, Phys. Lett. B 178, 416 (1986).

[39] M.Peskin, Nucl.Phys. B156 (1979) 365, G.Bhanot, Nucl.Phys. B156 (1979) 391 . D. Kharzeev and H. Satz, Nucl. Phys. A 590, 515C (1995).

[40] D. A. Appel, Phys. Rev. D 33, 717 (1986). J. P. Blaizot and L. D. McLerran, Phys. Rev. D 34, 2739 (1986). 
[41] M. Gyulassy and M. Plumer, Phys. Lett. B 243, 432 (1990). X. N. Wang, M. Gyulassy and M. Plumer, Phys. Rev. D 51, 3436 (1995) [arXiv:hepph/9408344]. G. Fai, G. G. Barnafoldi, M. Gyulassy, P. Levai, G. Papp, I. Vitev and Y. Zhang, arXiv:hep-ph/0111211.

[42] R. Baier, Y. L. Dokshitzer, S. Peigne and D. Schiff, Phys. Lett. B 345, 277 (1995) [arXiv:hep-ph/9411409]. R. Baier, Y. L. Dokshitzer, A. H. Mueller and D. Schiff, JHEP 0109, 033 (2001) [arXiv:hep-ph/0106347].

[43] E.V.Shuryak and I.Zahed, E. V. Shuryak and I. Zahed, arXiv:hepph/0207163. Submitted to Phys.Rev.D.

[44] E. V. Shuryak, Phys. Rev. C 66, 027902 (2002) arXiv:nucl-th/0112042.

[45] STAR Collaboration (C. Adler et al.) Phys.Rev.C66:034904,2002, e-Print Archive: nucl-ex/0206001

[46] J. Casalderry and E.Shuryak, in progress.

[47] L.Landau and E.Lifshitz, Fluid dynamics.

[48] D. Teaney, arXiv:nucl-th/0209024.

[49] L.Stodolsky,Phys.Rev.Lett. 75 (1995) 1044, E.V.Shuryak,Phys.Lett.B423 (1998) 9 .

[50] M. Stephanov, K. Rajagopal and E. V. Shuryak, Phys. Rev. Lett. 81, 4816 (1998) [arXiv:hep-ph/9806219]. Phys. Rev. D 60, 114028 (1999) [arXiv:hep$\mathrm{ph} / 9903292]$.

[51] M.Asakawa, U. W. Heinz, B.Muller, Phys.Rev.Lett.85:2072-2075,2000; hep-ph/0003169; S. Jeon, V. Koch, Phys.Rev.Lett.85:2076-2079,2000; hep$\mathrm{ph} / 0003168$

[52] H. Appelshauser et al. [NA49 Collaboration], Phys. Lett. B 459, 679 (1999) [arXiv:hep-ex/9904014].

[53] E.V.Shuryak,Talks at CERN Workshop on Event-by-Event fluctuations in heavy ion collisions, June 2001.

[54] S. Mrowczynski and E. V. Shuryak, arXiv:nucl-th/0208052. 


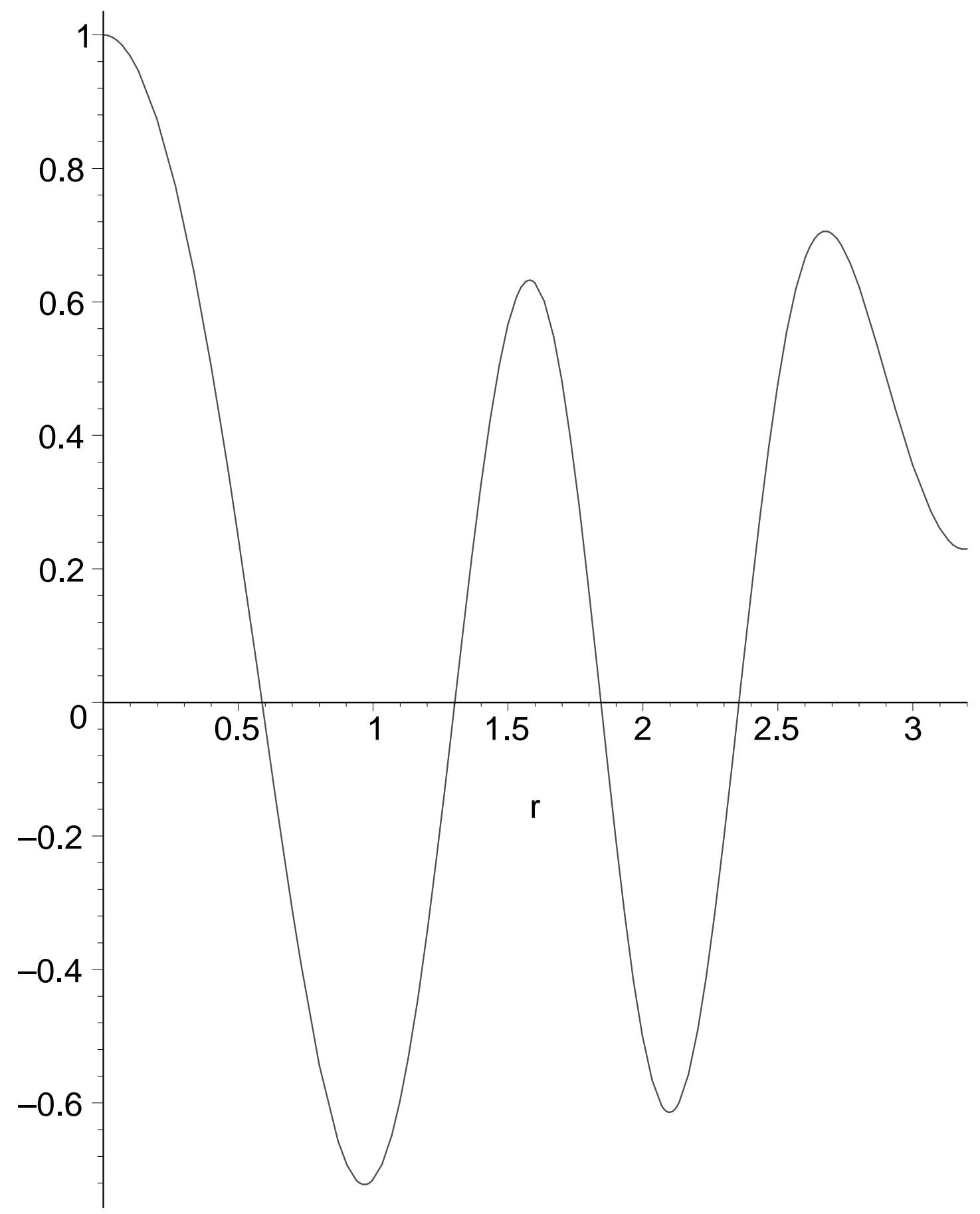

